\title{
Type 2 diabetes mellitus and myocardial ischemic preconditioning in symptomatic coronary artery disease patients
}

Paulo Cury Rezende, Rosa Maria Rahmi, Augusto Hiroshi Uchida, Leandro Menezes Alves da Costa, Thiago Luis Scudeler, Cibele Larrosa Garzillo, Eduardo Gomes Lima, Carlos Alexandre Wainrober Segre, Priscyla Girardi, Myrthes Takiuti, Marcela Francisca Silva, Whady Hueb*, Jose Antonio Franchini Ramires and Roberto Kalil Filho

\begin{abstract}
Background: The influence of diabetes mellitus on myocardial ischemic preconditioning is not clearly defined. Experimental studies are conflicting and human studies are scarce and inconclusive.

Objectives: Identify whether diabetes mellitus intervenes on ischemic preconditioning in symptomatic coronary artery disease patients.
\end{abstract}

Methods: Symptomatic multivessel coronary artery disease patients with preserved systolic ventricular function and a positive exercise test underwent two sequential exercise tests to demonstrate ischemic preconditioning. Ischemic parameters were compared among patients with and without type 2 diabetes mellitus. Ischemic preconditioning was considered present when the time to $1.0 \mathrm{~mm} \mathrm{ST} \mathrm{deviation} \mathrm{and} \mathrm{rate} \mathrm{pressure-product} \mathrm{were} \mathrm{greater} \mathrm{in} \mathrm{the} \mathrm{second}$ of 2 exercise tests. Sequential exercise tests were analyzed by 2 independent cardiologists.

Results: Of the 2,140 consecutive coronary artery disease patients screened, 361 met inclusion criteria, and 174 patients ( $64.2 \pm 7.6$ years) completed the study protocol. Of these, 86 had the diagnosis of type 2 diabetes. Among diabetic patients, 62 (72\%) manifested an improvement in ischemic parameters consistent with ischemic preconditioning, whereas among nondiabetic patients, 60 (68\%) manifested ischemic preconditioning ( $p=0.62)$. The analysis of patients who demonstrated ischemic preconditioning showed similar improvement in the time to $1.0 \mathrm{~mm}$ ST deviation between diabetic and nondiabetic groups (79.4 \pm 47.6 vs $65.5 \pm 36.4 \mathrm{~s}$, respectively, $p=0.12$ ). Regarding rate pressure-product, the improvement was greater in diabetic compared to nondiabetic patients (3011 \pm 2430 vs $2081 \pm 2139 \mathrm{bpm} \times \mathrm{mmHg}$, respectively, $p=0.01$ ).

Conclusions: In this study, diabetes mellitus was not associated with impairment in ischemic preconditioning in symptomatic coronary artery disease patients. Furthermore, diabetic patients experienced an improvement in this significant mechanism of myocardial protection.

Keywords: Ischemic preconditioning, Myocardial ischemia, Coronary artery disease, Diabetes mellitus

\footnotetext{
* Correspondence: whady.hueb@incor.usp.br

Department of Atherosclerosis, Heart Institute (InCor) of the University of São Paulo, Av. Dr. Eneas de Carvalho Aguiar 44, AB, Sala 114, 05403-000 Cerqueira César, São Paulo, Brazil
} 


\section{Background}

According to the World Health Organization, diabetes mellitus (DM) is one of the most common chronic diseases in the world, affecting $9.3 \%$ of all adults older than 25 years [1]. Moreover, the prevalence of diabetes has been substantially increasing during recent decades [2]. Diabetes poses a high risk for the development of cardiovascular diseases (CVD), because it heralds an accelerated process of atherosclerosis and an increased risk for atherothrombotic complications [3]. CVDs are the leading causes of death in diabetic patients [4] and the substantial rise in diabetes prevalence will ultimately lead to an increase in the burden of diabetes-related CVD in coming decades.

Among the cardiovascular diseases, ischemic heart disease is the most frequent $[5,6]$ and is associated with the highest morbidity and mortality in patients with type 2 DM.

Despite the advances in the treatment of coronary artery disease (CAD), morbidity and mortality are still high, especially in patients with type $2 \mathrm{DM}$ [7]. Moreover, despite the evolution in the treatment of hyperglycemia, patients with both CAD and diabetes have worse clinical outcomes, irrespective of the treatment applied [8].

Although DM alters the healthy functioning of arteries [9] and blood compounds [10], some clinical studies suggest that myocardial responses to ischemic insults may be deficient in diabetic patients $[11,12]$, leading to higher myocardial damage and risk of complications. Thus, recent studies [13] have focused on the understanding of such myocardial responses, aiming at discovering the mechanisms of myocardial protection to achieve less aggression and a better prognosis.

A substantial effort has been put forth to investigate any promising cardioprotective strategy to effectively reduce myocardial infarct size. And this matter is of particular importance to diabetic CAD patients [14]. By 1986, in a landmark experimental study, Murry and colleagues demonstrated that a short antecedent period of ischemia could result in a great reduction in myocardial infarct size [15]. For the first time, a method for reducing myocardial cell death other than reperfusion had been discovered. Since its discovery, this phenomenon, termed ischemic preconditioning (IP) has been extensively studied.

Currently, IP is the intrinsic myocardial mechanism that provides the greatest protection regarding the reduction in myocardial ischemic damage. It has demonstrated a $75 \%$ reduction in the infarcted area in animal models that have undergone the preconditioning protocol [15].

IP is assumed as a mechanism of myocardial protection in which brief episodes of sublethal myocardial ischemia followed by reperfusion trigger multiple intracellular pathways that ultimately result in greater myocardial resistance to a subsequent intense ischemic injury. Experimentally, this phenomenon was demonstrated by the reduction in the infarcted area by short episodes of ischemia prior to a pronounced ischemic insult [15].

Although IP was initially thought to result from the opening of collateral vessels and higher coronary flow [16], some interesting studies have shown that the phenomenon occurs irrespective of coronary flow changes $[17,18]$. Such studies also demonstrated that IP can be observed by sequential exercise tests (SETs), in which the improvement in ischemic parameters in the second of 2 SETs were confirmed by invasive measurements of myocardial oxygen consumption. Moreover, the clinical observation that patients with chronic ischemic heart disease frequently describe attenuation or even cessation of angina symptoms if they rest and restart the exercise is termed "warm-up" or "walk-through" angina. This phenomenon has been related to IP and documented in studies using SETs [19, 20].

Following the demonstration of IP, the cellular mechanisms underlying the phenomenon began to be investigated. Although some cellular pathways have not been fully elucidated, there is a growing understanding of some phenomena and currently it is assumed that IP may be modulated by external factors like age [21], diseases [22-24], as well as some specific classes of medications [25-27].

Currently, the opening of K-ATP channels plays a fundamental role in the cellular cascades of IP [28]. It is also assumed that medications that bind to this channel may interfere with the IP mechanism. Thus, some classes of medications like the oral hypoglycemic agents may also block such channels in extrapancreatic sites, such as in the heart. It has been shown by some authors that these medications may cause the blockage of myocardial IP [29].

However, despite the fact that some oral hypoglycemic agents may interfere with IP, it is still uncertain whether the intrinsic, complex, intracellular alterations of diabetes itself may affect the cellular mechanisms of this cardioprotective phenomenon. Experimental studies aimed at studying this question have shown conflicting results [30]. Some of these studies have shown that diabetes does not interfere with IP [31, 32], but others have shown negative influences [33, 34]. The great variability in these results is especially due to differences in study protocols, most notably the variability in animal models studied, in the protocols to induce diabetes, the duration of the disease, as well as the variability in myocardial injury protocols. On the other hand, studies in humans are scarce and their results have also been conflicting $[12,13,35]$. Thus, in this study, we aimed at identifying IP in symptomatic multivessel CAD patients and compared the results among patients with and without type 2 DM.

\section{Methods}

\section{Study design}

This was a prospective study that included patients with symptomatic CAD followed by the Medicine, Angioplasty, 
or Surgery Study (MASS) research group. The study design and protocol have been published elsewhere [36]. Briefly, patients were enrolled who had angina symptoms, multivessel CAD, preserved left systolic ventricular function, and a recent, positive ischemic treadmill stress test. All patients gave written informed consent. The diagnosis of type 2 DM was based on American Diabetes Association guidelines diagnostic criteria [37]. Multivessel CAD was confirmed by the finding on cineangiocoronariography of atherosclerotic stenosis of at least $70 \%$ obstruction in 2 or more coronary artery territories. The systolic ventricular function was measured by transthoracic echocardiography and was considered preserved if ejection fraction was $\geq 0.50$. Treadmill exercise tests were considered positive for myocardial ischemia if a horizontal or downsloping ST-segment deviation was $\geq 1.0 \mathrm{~mm}$, associated or not with thoracic discomfort. Exclusion criteria were single-vessel CAD, left main CAD, impaired systolic ventricular function (defined as an ejection fraction $<0.50$ ), recent and negative treadmill exercise test, high-risk positive treadmill exercise test, limiting angina symptoms, an acute coronary syndrome in the prior 3 months, electrocardiographic signs that could make difficult the interpretation of ischemic changes (left bundle-branch block, ST-segment deviation), arrhythmias like atrial fibrillation or flutter, severe valvular disease, cardiomyopathies, or patient refusal to participate in this study. The Ethics Committee of the Heart
Institute approved this study. All procedures were in accordance with the terms of the Declaration of Helsinki.

\section{Patients}

After cardiologic evaluation, all patients were instructed to stop medications with cardiovascular properties 5 days before SETs. Diabetic patients were also instructed to stop antidiabetes medications 5 days before the tests. Only nitrates were allowed until $12 \mathrm{~h}$ before the tests. Patients were also recommended to not perform physical activities during the period without the use of medications and were under appropriate nutritional control. A telephone contact was available $24 \mathrm{~h}$ a day in case of questions or worsening symptoms. Before treadmill test initiation, a medical team reassessed the symptoms to ascertain clinical stability.

\section{Sequential treadmill exercise tests}

All patients underwent 2 SETs, symptom limited, with a 30-min interval between them. The modified Bruce protocol was applied. Tests were conducted during the same period each day, $1 \mathrm{~h}$ after lunchtime, on the same treadmill, (MAT 2100 treadmill and a Fukuda Denshi ML8000 Stress Test system (Fukuda Denshi; Bunkyo-ku, Tokyo, Japan).

A 12-lead electrocardiogram, heart rate, and arterial blood pressure were obtained with the patient in the

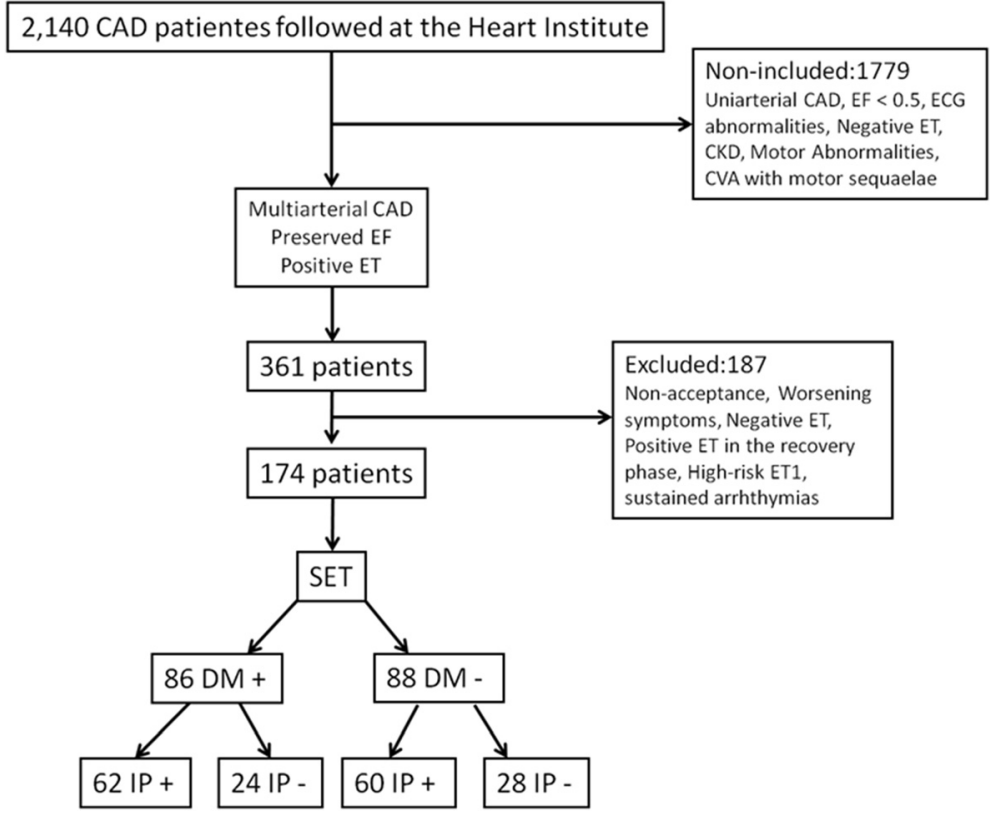

Fig. 1 Study flow chart. Of the 2,140 initial CAD patients screened, 361 met the inclusion criteria and were enrolled, and 174 completed the study protocol. The study population comprised 86 diabetic and 88 nondiabetic patients. $C A D=$ coronary artery disease; $C K D=$ chronic kidney disease; $C V A$ = cerebrovascular accident; $E C G=$ electrocardiogram; $E F=$ ejection fraction; $E T$ = exercise test; $D M=$ diabetes mellitus; IP = ischemic preconditioning; SET = sequential exercise test (s) 
Table 1 Main demographic, biochemical, and clinical characteristics of the study population

\begin{tabular}{|c|c|c|c|}
\hline Total $N=174$ & Diabetics $(n=86)$ & Non-diabetics $(n=88)$ & $p$ Value \\
\hline Age & $64.1 \pm 6.8$ & $64.3 \pm 8.6$ & 0.84 \\
\hline Male n (\%) & $73(84.9)$ & $76(86.3)$ & 0.83 \\
\hline Hypertension & $64(80)$ & $74(84.1)$ & 0.54 \\
\hline Smokers & $7(8.8)$ & 7 (8.6) & \\
\hline Previous smokers & $38(48.1)$ & $33(40.7)$ & 0.6 \\
\hline Non-smokers & $34(43.0)$ & $41(50.6)$ & \\
\hline Previous AMI & $36(46.1)$ & $17(22.7)$ & 0.004 \\
\hline CABG & $28(34.6)$ & $23(26.1)$ & 0.25 \\
\hline $\mathrm{PCl}$ & $24(30.4)$ & $28(32.2)$ & 0.87 \\
\hline EF & $0.61 \pm 0.06$ & $0.62 \pm 0.06$ & 0.2 \\
\hline Tri-vessel disease & $38(52)$ & $43(51.2)$ & 1.0 \\
\hline Bi-vessel disease & $35(48)$ & $41(48.8)$ & 1.0 \\
\hline LAD disease & $63(87.5)$ & $74(86.0)$ & 1.0 \\
\hline Collateral circulation & $34(50.7)$ & $38(46.9)$ & 0.64 \\
\hline Collateral grade $2 / 3$ & $30(88.2)$ & $32(84.2)$ & 0.74 \\
\hline Weight (kg) & $74.5 \pm 11.0$ & $73.2 \pm 12.7$ & 0.51 \\
\hline Height (m) & $1.66 \pm 8.1$ & $1.66 \pm 9.1$ & 0.75 \\
\hline BMI & $26.5 \pm 2.8$ & $26.4 \pm 3.2$ & 0.54 \\
\hline Fasting glycemia & $143.5 \pm 47.0$ & $99.0 \pm 9.4$ & 0.0001 \\
\hline $\mathrm{A} 1 \mathrm{C}$ & $7.35 \pm 1.61$ & $5.63 \pm 0.30$ & 0.0001 \\
\hline BUN & $41.9 \pm 13.4$ & $40.1 \pm 9.4$ & 0.32 \\
\hline Creatinine & $1.04 \pm 0.23$ & $1.07 \pm 0.20$ & 0.37 \\
\hline Total cholesterol & $156.5 \pm 34.5$ & $168.9 \pm 36.3$ & 0.02 \\
\hline LDL cholesterol & $90.5 \pm 27.4$ & $102.0 \pm 32.7$ & 0.01 \\
\hline HDL cholesterol & $38.0 \pm 9.6$ & $40.3 \pm 9.0$ & 0.12 \\
\hline Triglycerides & $147.2 \pm 88.3$ & $132.6 \pm 72.3$ & 0.24 \\
\hline
\end{tabular}

Data are expressed as means \pm standard deviation or as absolute and relative risks

$A M I$ stands for acute myocardial infarction, $C A B G$ coronary artery bypass surgery, $P C l$ percutaneous coronary intervention, $E F$ ejection fraction, $L A D$ left anterior descending, $B M I$ body mass index, $A 1 C$ glycosylated hemoglobin, $B U N$ blood urea nitrogen, $L D L$ low-density lipoprotein, $H D L$ high-density lipoprotein

standing position at baseline. A 12-lead electrocardiogram was also obtained at each 1.0-min interval during exercise, at peak exercise, each minute up to 5 min during the recovery phase, at the onset of $1.0 \mathrm{~mm}$ ST-segment depression, during arrhythmias, and when it was clinically relevant.

A computer-assisted electrocardiogram was monitored during the exercise and recovery phases. The level of the
ST-segment deviation was based on visual assessment of the $0.08 \mathrm{~s}$ after the J point by 2 independent cardiologists. In case of disagreement, a third cardiologist was consulted, and the matter was resolved by consensus. Only the horizontal or downsloping ST-segment deviations were considered for the time to onset of $1.0-\mathrm{mm}$ ST-segment depression evaluation $(\mathrm{T}-1.0 \mathrm{~mm})$. Criteria for interrupting the exercise test were ST-segment depression $\geq 3.0 \mathrm{~mm}$, ST segment elevation $\geq 2.0 \mathrm{~mm}$, maximum age-related heart rate, severe arterial hypotension or hypertension, severe chest pain, physical exhaustion, and sustained arrhythmias.

The following parameters were systematically measured: resting heart rate and arterial blood pressure, heart rate and arterial blood pressure at peak exercise, $\mathrm{T}-1.0 \mathrm{~mm}$ in seconds, rate pressure product (RPP) at the onset of T-1.0 mm, and exercise duration in seconds.

The improvement in ischemic parameters $(\mathrm{T}-1.0 \mathrm{~mm}$ and RPP) in the second exercise test compared to the first one indicated the presence of IP. Both tests were blinded for the cardiologist's analysis.

Other parameters recorded during SETs were the total exercise time, the occurrence and density of supraventricular and ventricular arrhythmias, and ST-segment deviation morphology, during exercise and recovery phases. Both arrhythmias and ST-segment morphology were graded according to their density and severity.

According to the levels of fasting glycemia and glycosylated hemoglobin from baseline, patients were separated into quartiles. For fasting glycemia, the quartiles were defined as Quartile 1: $<90 \mathrm{mg} / \mathrm{dL}$, Quartile 2: $90-100 \mathrm{mg} /$ $\mathrm{dL}$, Quartile 3: 101-125 mg/dL, and Quartile 4: $\geq 126 \mathrm{mg} /$ $\mathrm{dL}$. For glycosylated hemoglobin, the quartiles were defined as Quartile 1: $<5.7 \%$, Quartile 2: 5.7-6.3 \%, Quartile 3: 6.4-6.9\%, and Quartile 4: $\geq 7.0 \%$. The prevalence of patients who expressed IP was compared among the quartiles.

\section{Sample size calculation}

The sample size calculation has been previously published elsewhere [36]. Briefly, it was determined by the analysis of studies with similar methodologies that included only diabetic patients [38] and studies that included only nondiabetic patients [21]. By the differences in $\mathrm{T}-1.0 \mathrm{~mm}$ in diabetic and nondiabetic patients, and accounting for an expected loss of $30 \%$ of patients who do not demonstrate the IP phenomenon (estimated

Table 2 Classes of medications used by the two groups of diabetic and nondiabetic patients

\begin{tabular}{lllllllll}
\hline & Aspirin / Clopidogrel & Statins & Beta-blockers & ACEl / ARB & Calcium blockers & Diuretics & OAD & Insulins \\
\hline DM & $93.2 \%$ & $93.2 \%$ & $89.8 \%$ & $78.4 \%$ & $35.2 \%$ & $37.5 \%$ & $88.5 \%$ & $26.5 \%$ \\
Non-DM & $96.5 \%$ & $96.5 \%$ & $86.2 \%$ & $78.2 \%$ & $36.8 \%$ & $34.5 \%$ & - & - \\
\hline
\end{tabular}

$D M$ stands for diabetic patients, Non-DM nondiabetic patients, $A C E I$ angiotensin converting enzyme inhibitors, $A R B$ angiotensin receptor blockers, $O A D$ oral antidiabetic drugs 

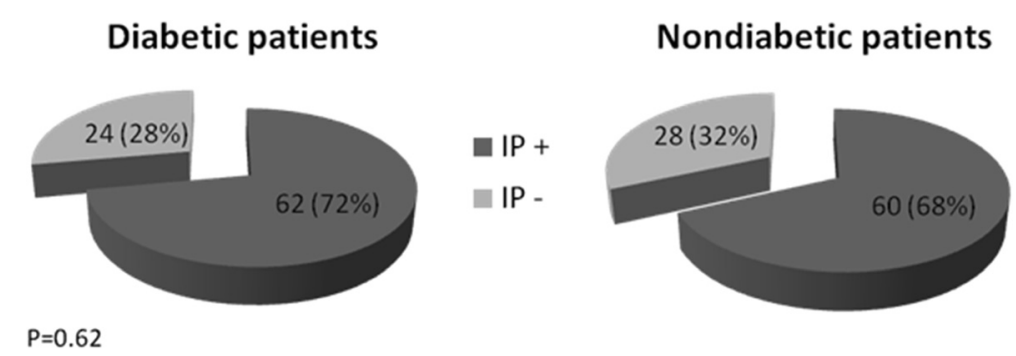

Fig. 2 Pie charts showing the number and percentage of diabetic and nondiabetic patients who demonstrated IP. IP =ischemic preconditioning

based on the experience of our research group), 70 patients with diabetes and 70 without diabetes should be included to test the null hypothesis that the population means were similar (power 0.9 and alpha error 0.05).

\section{Statistical analysis}

Continuous variables were compared using an unpaired Student's $t$ test or Mann-Whitney test, when appropriate. The Kolmogorov-Smirnov test was used to assess the distribution of continuous variables. Discrete variables were compared using chi-squared test. Fisher's test was used when appropriate. Data are expressed as mean \pm standard deviation or as absolute frequencies and percentages. The software SPSS, version 17.0, was used for all statistical analyses. All tests were 2 -sided, and a value of $p<0.05$ was considered significant.

\section{Results}

Of 2,140 patients with stable CAD followed at our institution, 361 met the inclusion criteria. Of those, $187 \mathrm{did}$ not complete the study protocol. The main reasons for non-inclusion and exclusion are shown in Fig. 1. Thus, a total of 174 patients completed the 2 SETs and had IP assessed. The study population comprised 86 patients with DM and 88 without this diagnosis (Fig. 1).

The main demographic, clinical and biochemical characteristics of the 2 groups are presented in Table 1. Despite

Table 3 T-1.0 $\mathrm{mm}$ in exercise test 1 (ET1) and exercise test 2 (ET2) and the difference between the 2 tests (ET2-ET1) in diabetic and nondiabetic patients who demonstrated IP (IP+) or who did not demonstrate IP (IP-)

\begin{tabular}{lllll}
\hline & ET1 & ET2 & ET2-ET1 & $p$ Value* \\
\hline DM & $269.2 \pm 117.8$ & $320.9 \pm 132.3$ & $51.7 \pm 63.2$ & 0.15 \\
Non-DM & $275.6 \pm 111.9$ & $314.6 \pm 126.2$ & $39.0 \pm 52.3$ & \\
DM / IP+ & $274.8 \pm 102.8$ & $354.3 \pm 115.1$ & $79.4 \pm 47.6$ & 0.12 \\
Non-DM / IP+ & $296.9 \pm 107.7$ & $362.4 \pm 108.5$ & $65.5 \pm 36.4$ & \\
DM / IP - & $254.7 \pm 151.6$ & $234.7 \pm 137.0$ & $-20.0 \pm 36.4$ & 0.80 \\
Non-DM / IP - & $230.1 \pm 108.7$ & $212.4 \pm 98.2$ & $-17.7 \pm 31.9$ &
\end{tabular}

Data are expressed as means \pm standard deviation. $D M$ stands for diabetes mellitus, IP + ischemic preconditioning present, IP - ischemic preconditioning absent, $E T$, exercise test. ${ }^{*}$ The $p$ values are from the comparison of ET2-ET1 among diabetic and nondiabetic patients the higher prevalence of previous myocardial infarction in the diabetic population and lipid profile (higher LDLcholesterol levels in nondiabetic patients), both groups had homogeneous characteristics. The duration of diabetes was $11.5 \pm 8.8$ years $($ mean $\pm S D)$, with a median of 10 years (interquartiles ranges of 6 and 15 years). Table 2 shows the medications used in the two groups of patients.

\section{Demonstration of IP}

Among the 86 diabetic patients, 62 (72\%) had an improvement in T-1.0 mm consistent with IP. Among the 88 nondiabetic patients, 60 (68\%) had an ischemic improvement consistent with IP (Fig. 2, $p=0.62$ ).

The T-1.0 mm results demonstrated that diabetic patients who demonstrated IP had an improvement in T-1.0 mm between the 2 SETs of $79.4 \pm 47.6 \mathrm{~s}$, whereas nondiabetic patients who demonstrated IP had an improvement of $65.5 \pm 36.4 \mathrm{~s}$ (Table 3, $p=0.12$ ).

Regarding RPP, the group of diabetic patients who demonstrated IP had an improvement of 3,011 \pm 2,430 bpm $\times \mathrm{mmHg}$, whereas nondiabetic patients had an improvement of 2,081 $\pm 2,139 \mathrm{bpm} \times \mathrm{mmHg}$ (Table 4, $p=0.01$ ).

Table 5 shows the hemodynamic parameters heart rate and blood pressure at baseline and peak exercise in the 4 groups of patients.

Table 4 RPP in exercise test 1 (ET1) and exercise test 2 (ET2) and the difference between the 2 tests (ET2-ET1) in diabetic and nondiabetic patients who demonstrated IP (IP+) or who did not demonstrate IP (IP-)

\begin{tabular}{lllll}
\hline & $E T 1$ & $E T 2$ & $E T 2-E T 1$ & $\begin{array}{l}p \\
\text { Value }\end{array}$ \\
\hline DM / IP+ & $22,841 \pm$ & $25,853 \pm$ & $3,011 \pm 2,430$ & 0.01 \\
& 4,500 & 4,742 & & \\
Non-DM / IP & $23,094 \pm$ & $25,175 \pm$ & $2,081 \pm 2,139$ & \\
+ & 5,312 & 5,485 & & \\
DM / IP - & $22,705 \pm$ & $22,124 \pm$ & $-580 \pm 2,250$ & 0.43 \\
& 4,000 & 3,686 & & \\
Non-DM / IP - & $23,910 \pm$ & $22,869 \pm$ & $-1,050 \pm$ & \\
& 5,380 & 5,351 & 2,027 & \\
\hline
\end{tabular}

Data are expressed as means \pm standard deviation. $D M$ stands for diabetes mellitus, IP + ischemic preconditioning present, IP - ischemic preconditioning absent, ET exercise test. * The $p$ values are from the comparison of ET2-ET1 among diabetic and nondiabetic patients 
Table 5 Hemodynamic parameters, heart rate and blood pressure in the 4 groups of patients, diabetic and nondiabetic, according to the demonstration of IP

\begin{tabular}{lllll}
\hline & Variables & ET 1 & ET 2 & ET2-ET1 \\
\hline DM / IP + & HR (baseline) & $79.0 \pm 15.1$ & $86.3 \pm 17.0$ & $7.3 \pm 11.0$ \\
& HR (peak) & $139.0 \pm 15.1$ & $142.1 \pm 14.7$ & $3.2 \pm 5.6$ \\
& BP (baseline) & $156.5 \pm 18.2$ & $149.7 \pm 19.3$ & $-6.8 \pm 12.6$ \\
DM / IP - & BP (peak) & $192.0 \pm 25.0$ & $191.8 \pm 23.9$ & $-0.16 \pm 15.4$ \\
& HR (baseline) & $79.0 \pm 14.7$ & $84.4 \pm 14.9$ & $5.4 \pm 8.0$ \\
& HR (peak) & $141.7 \pm 14.3$ & $143.0 \pm 13.5$ & $1.3 \pm 7.4$ \\
Non-DM / IP + & BP (baseline) & $153.3 \pm 22.6$ & $147.1 \pm 19.7$ & $-6.2 \pm 14.7$ \\
& HP (peak) & $183.7 \pm 23.2$ & $180.6 \pm 22.2$ & $-3.12 \pm 14.7$ \\
& HR (paseline) & $78.0 \pm 13.1$ & $87.1 \pm 13.9$ & $9.1 \pm 7.9$ \\
& BP (baseline) & $139.6 \pm 15.4$ & $144.3 \pm 14.7$ & $4.7 \pm 5.7$ \\
& BP (peak) & $193.5 \pm 23.6$ & $190.2 \pm 24.5$ & $-3.3 \pm 12.4$ \\
Non-DM / IP - & HR (baseline) & $82.5 \pm 12.9$ & $87.1 \pm 12.6$ & $4.6 \pm 7.1$ \\
& HR (peak) & $139.3 \pm 12.4$ & $139.6 \pm 13.0$ & $0.35 \pm 5.2$ \\
& BP (baseline) & $158.6 \pm 26.2$ & $147.9 \pm 26.1$ & $-10.7 \pm 14.6$ \\
& BP (peak) & $204.6 \pm 25.3$ & $194.8 \pm 28.1$ & $-8.9 \pm 16.5$ \\
\hline
\end{tabular}

Data are expressed as means \pm standard deviation. DM stands for diabetic patients, Non-DM nondiabetic patients, IP + ischemic preconditioning present, $I P$ - ischemic preconditioning absent, $E T$ exercise test

\section{Analyses of secondary ergometric variables}

The improvement in the total exercise time comparing the 2 SETs was similar between the groups of diabetic and nondiabetic patients $(20 \pm 39 \mathrm{~s}$ versus $17 \pm 36 \mathrm{~s}$, respectively, $p=0.60$ ).

The improvement in the frequency and severity of arrhythmias had similar results among diabetic and nondiabetic patients who experienced IP (50\% versus $63 \%$ of patients demonstrated improvement in arrhythmias, respectively; $p=0.41$ ).

The improvement in the ST-segment deviation morphology was also similar among diabetic and nondiabetic patients (38.5\% versus $48.3 \%$, respectively, $p=0.41)$.

\section{Analysis of glycemic variables}

When the total group of patients $(n=174)$ was stratified according to the demonstration of IP, the frequency of diabetic patients as well as the levels of fasting glycemia and glycosylated hemoglobin were similar between the 2 groups, as shown in Table 6.

In addition, when we stratified patients by quartiles of glycemia and glycosylated hemoglobin, there was no statistical difference in terms of IP demonstration among the different quartiles (Fig. 3).

\section{Discussion}

Considering that diabetes is an independent risk factor for the occurrence of major cardiovascular events and
Table 6 Frequency of diabetes mellitus, and levels of fasting glycemia and A1C in the study population according to the expression of IP

\begin{tabular}{llll}
\hline & $\mathrm{IP}+(n=122)$ & $\mathrm{IP}-(n=52)$ & $P$ Value \\
\hline $\mathrm{DM} n(\%)$ & $62(50.8)$ & $24(46.1)$ & 0.57 \\
Fasting glycemia & $121.3 \pm 42.6$ & $118.9 \pm 34.1$ & 0.72 \\
A1C & $6.63 \pm 1.6$ & $6.32 \pm 1.2$ & 0.25 \\
\hline
\end{tabular}

Data are expressed as means \pm standard deviation and as absolute and relative frequencies $D M$ stands for diabetes mellitus, $A 1 c$ glycosylated hemoglobin, $I P+$ ischemic preconditioning present, IP - ischemic preconditioning absent

mortality $[5,6]$, it is reasonable to consider that diabetes might damage the protective mechanism of IP in CAD patients, leading to worse outcomes. However, in this study, the presence of type 2 DM did not have any deleterious effects on the myocardial protective mechanism termed ischemic preconditioning.

In this context, our study showed that patients with type 2 DM demonstrated IP in similar frequency and intensity compared with nondiabetic patients and, interestingly, our data indicated an improvement in ischemic parameters associated with diabetes. The analyses of the data showed a better ischemic response evaluated by RPP in diabetic patients. Thus, this study adds clinical information on some questions that have emerged from contradictory experimental studies.

The improvement in myocardial oxygen consumption, observed by the analysis of RPP, was more pronounced in patients with compared to those without diabetes, indicating better adaptation of the myocardium of diabetic patients after an ischemic insult. Moreover, diabetic patients had an improvement in T-1.0 $\mathrm{mm}$ greater than that in nondiabetic patients, although it did not reach statistical significance. Analyzes of the total exercise time also confirmed the main results of the study, as times were similar among patients with and without DM. Similarly, the ST-segment deviation morphology did not differ among diabetic and nondiabetic patients.

Because myocardial ischemia is a frequent cause of arrhythmias during treadmill stress tests, we also assessed the occurrence and complexity of arrhythmias and their improvement during SET. Thus, the improvement in the occurrence of arrhythmias also confirmed the main findings of the study, as the occurrence did not differ among diabetic and nondiabetic patients.

Interestingly, analyzes of the percentage of patients who demonstrated IP among different quartiles of fasting glycemia and glycosylated hemoglobin showed similar rates of IP demonstration. Thus, despite differences in glycemic control, patients demonstrated IP in similar proportions. This analyzes infer that the differences in the intensity of glycemic control in our population did not prevent the demonstration of this cardioprotective phenomenon. 

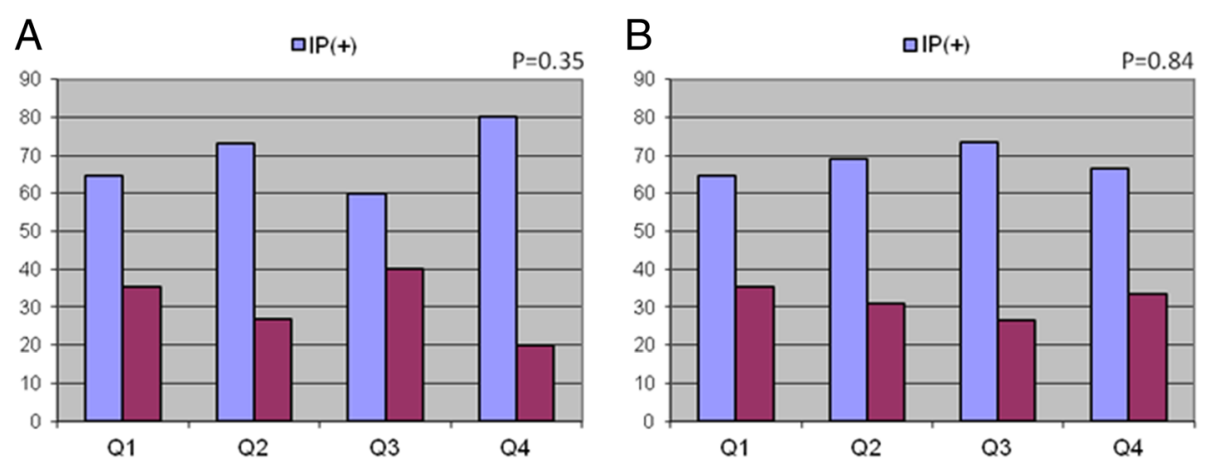

Fig. 3 Graphs showing the percentage of patients who demonstrated ischemic preconditioning (IP+ in blue) and who did not demonstrate ischemic preconditioning (IP - in red) stratified into quartiles of A1c (graph a) and Fasting Glycemia (graph b). IP = ischemic preconditioning; $\mathrm{Q}=$ quartile (s). $\mathrm{X}$ axis represents the percentage of patients and $\mathrm{Y}$ axis the quartiles of A1c and Fasting Glycemia

However, despite our consistent findings, the information from this study is contrary to Lee's et al. [11] and Ishihara's et al. [12], who have also studied IP in humans.

Lee et al. [11] studied diabetic and nondiabetic CAD patients during percutaneous coronary interventions, and evaluated the action of hypoglycemic agents on IP. They enrolled patients with CAD and formal indication for coronary intervention. Patients underwent consecutive balloon coronary inflations. During the second sequential balloon inflation, patients had less thoracic discomfort, less myocardial lactate production, and lower ST-segment deviation. Moreover, the authors observed that diabetic patients treated with glimepiride had higher lactate production compared to nondiabetic patients treated with glimepiride. Despite this finding, an important limitation of this study is that there was not a direct comparison of diabetic and nondiabetic patients who had no drug interference in IP evaluation.

Ishihara et al. [12] studied patients hospitalized due to an acute myocardial infarction and evaluated the effects of preinfarct angina, on the release of cardiac markers of necrosis, ventricular function, and in-hospital death and compared the results among diabetic and nondiabetic patients. The authors found that in the nondiabetic population, patients with preinfarct angina had a lower release of cardiac markers of necrosis, better recovery of ventricular function, and lower in-hospital mortality, compared to patients with no preinfarct angina. On the other hand, when they analyzed diabetic patients, these variables were not different among patients with and without preinfarct angina. Thus, the authors inferred that diabetes prevented the appearance of IP. Despite their findings, Ishihara's study has important limitations. First, this was a retrospective study, which included a small number of diabetic $(n=121)$ compared to nondiabetic patients $(n=490)$. In addition, among the 121 diabetic patients, 53 were taking hypoglycemic medications. Many studies have shown that some of these drugs may block IP [39], and it has been speculated that this interference with IP mechanisms may partially explain the worse prognosis of diabetic patients hospitalized due to an acute myocardial infarction [40].

On the other hand, other experimental studies that evaluated "in vitro" human myocardial tissue [41, 42] showed results that match those of the present study.

Ghosh et al. [41] studied human atrial appendages from diabetic and nondiabetic patients after a severe ischemic insult. They evaluated the release of cardiac biomarkers of necrosis and the percentage of tissue viability. Among other findings, the authors found a similar intensity in myocardial protection among diabetic and nondiabetic patients.

Additionally, Cleveland et al. [42] evaluated the contractile function of isolated right atrial trabeculae from CAD patients, resected during coronary artery bypass graft surgery. The authors observed the improvement in the contractile strength after an ischemic insult. They showed that the diabetic group that underwent the preconditioning stimuli had similar improvement in contractile function compared to the nondiabetic group.

Moreover, a small study conducted by Bilinska and colleagues [35] with diabetic patients treated with glibenclamide, gliclazide, and diet compared the demonstration of IP in these groups with that in nondiabetic patients. Besides the main findings of the study, which were related to the effects of sulfonylureas in the warmup phenomenon, they showed that the group of diabetic patients on diet $(n=15)$ had similar improvement in ischemic parameters compared to nondiabetic patients $(n=17)$. Of note, this was a secondary result, and the small sample size did not permit to make a definitive conclusion.

\section{Study limitations and strengths}

This prospective study on IP in humans had a sample size powered enough to compare IP demonstration in 2 
populations with matched clinical characteristics and evaluated the possible interference of diabetes on a myocardial protective mechanism. However, some reflection on the main findings is necessary. In this study, the group of patients with diabetes was under strict control of hyperglycemia, and this is observed by the controlled levels of fasting glucose and glycosylated hemoglobin in the group of diabetic patients. Assuming that hyperglycemia may interfere negatively with IP [43], it is possible that the result of this study would be different in a population under less strict hyperglycemic control.

\section{Clinical practice perspectives}

Patients with symptomatic myocardial ischemia evolve with diverse prognosis. In this context, the response of the myocardium to an ischemic insult may probably play an important role in prognosis. One mechanism that may interfere with such a prognosis may be the presence of a protective myocardial phenomenon, termed ischemic preconditioning. Because diabetes is an independent risk factor for the occurrence of major cardiovascular events, it is reasonable that it may compromise this cardioprotective mechanism, leading to worse outcomes. Contrary to the initial expectation, the analysis of our data revealed that diabetic patients showed this protective phenomenon similarly to nondiabetic patients. Moreover, diabetic patients had an improvement in hemodynamic parameters. This study permits to demystify that diabetes interferes with myocardial responses to ischemic insults.

\section{Conclusions}

In this study, diabetes mellitus did not substantially affect myocardial IP in symptomatic CAD patients. Furthermore, diabetic patients experienced an improvement in this significant mechanism of myocardial protection.

\section{Abbreviations \\ CAD: Coronary artery disease; CVD: Cardiovascular disease; DM: Diabetes mellitus; IP: Ischemic preconditioning; MASS: Medicine, Angioplasty, or Surgery Study; RPP: Rate-pressure product; SET: Sequential exercise test; $\mathrm{T}-1.0 \mathrm{~mm}$ : Time to $1.0 \mathrm{~mm}$ ST deviation.}

\section{Competing interests}

The authors declare that they have no competing interests.

\footnotetext{
Authors' contributions

Each one of the authors have performed the following contributions to this work: P.C.R. researched data and wrote the manuscript. R.M.R. and A.H.U. contributed to exercise treadmill tests and drafting of the manuscript. L.M.A.C., T.L.S., C.L.G. and E.G.L. contributed to exercise treadmill tests and data interpretation. C.A.W.S., P.G., M.T. and M.F.S. contributed to patient selection and reviewed the manuscript. W.H. conceived this study, contributed to discussion, and reviewed the manuscript. J.A.F.R. and R.K.F. reviewed the manuscript. W.H. Was the responsible for the final approval of the manuscript submitted. All authors read and approved the final manuscript.
}

\section{Acknowledgments}

We would like to thank all members of the MASS Research team for their hard work in this study. This study was funded by the Zerbini Foundation. Medical writing support was provided by Ann Conti Morcos.

Received: 6 April 2015 Accepted: 21 May 2015

Published online: 30 May 2015

\section{References}

1. Global status report on noncommunicable diseases 2010. Geneva: World Health Organization. 2011. Available at: http://www.who.int/nmh/ publications/ncd_report2010. Accessed 29 January, 2015.

2. Wild S, Roglic G, Green A, Sicree R, King H. Global prevalence of diabetes: estimates for the year 2000 and projections for 2030. Diabetes Care. 2004;27:1047-53.

3. Beckman JA, Creager MA, Libby P. Diabetes and atherosclerosis: epidemiology, pathophysiology, and management. JAMA. 2002;287:2570-81.

4. Roglic G, Unwin N, Bennett PH, Mathers C, Tuomilehto J, Nag S, et al. The burden of mortality attributable to diabetes: realistic estimates for the year 2000. Diabetes Care. 2005;28:2130-5.

5. Haffner SM, Lehto S, Ronnemaa T, Pyorala K, Laakso M. Mortality from coronary heart disease in subjects with type 2 diabetes and in nondiabetic subjects with and without prior myocardial infarction. N Engl J Med. 1998;339:229-34.

6. Almdal T, Scharling H, Jensen JS, Pyorala K, Laakso M. The independent effect of type 2 diabetes mellitus on ischemic heart disease, stroke, and death: a population-based study of 13,000 men and women with 20 years of follow-up. Arch Intern Med. 2004;164:1422-6.

7. Myers WO, Blackstone EH, Davis K, Foster ED, Kaiser GC. CASS registry long-term surgical survival. Coronary artery surgery study. J Am Coll Cardiol. 1999;33:488-98.

8. Lima EG, Hueb W, Garcia RMR, Pereira AC, Soares PR, Favarato D, et al. Impact of diabetes on 10-year outcomes of patients with multivessel coronary artery disease in the Medicine, Angioplasty, or Surgery Study ॥ (MASS II) trial. Am Heart J. 2013;166:250-7.

9. Williams SB, Cusco JA, Roddy MA, Johnstone MT, Creager MA. Impaired nitric oxide-mediated vasodilation in patients with non-insulin-dependent diabetes mellitus. J Am Coll Cardiol. 1996;27:567-74.

10. Vinik Al, Erbas T, Park TS, Nolan R, Pittenger GL. Platelet dysfunction in type 2 diabetes. Diabetes Care. 2001;24:1476-85.

11. Lee TM, Chou TF. Impairment of myocardial protection in type 2 diabetic patients. J Clin Endocrinol Metab. 2003:88:531-7.

12. Ishihara M, Inoue I, Kawagoe T, Shimatani Y, Kurisu S, Nishioka K, et al. Diabetes mellitus prevents ischemic preconditioning in patients with a first acute anterior wall myocardial infarction. J Am Coll Cardiol. 2001;38:1007-11.

13. Yellon DM, Hausenloy DJ. Myocardial reperfusion injury. N Engl J Med. 2007;357:1121-35.

14. Wider J, Pryklenk K. Ischemic conditioning: the challenge of protecting the diabetic heart. Cardiovasc Diagn Ther. 2014;4:383-96.

15. Murry CE, Jennings RB, Reimer KA. Preconditioning with ischemia: a delay of lethal cell injury in ischemic myocardium. Circulation. 1986;74:1124-36.

16. Jaffe MD, Quinn NK. Warm-up phenomenon in angina pectoris. Lancet. 1980;2:934-6.

17. Okazaki Y, Kodama K, Sato H, Kitakaze M, Hirayama A, Mishima M, et al. Attenuation of increased regional myocardial oxigen consumption during exercise as a major cause of warm-up phenomenon. J Am Coll Cardiol. 1993;21:1597-604.

18. Williams DO, Bass TA, Gewirtz H, Most AS. Adaptation to the stress of tachycardia in patients with coronary artery disease: insight into the mechanism of the warm-up phenomenon. Circulation. 1985;71:687-92.

19. Ylitalo K, Jama L, Raatikainen P, Peuhkurinen K. Adaptation to myocardial ischemia during repeated dynamic exercise in relation to findings at cardiac catheterization. Am Heart J. 1996;131:689-97.

20. Maybaum S, llan M, Mogilevsky J, Tzivoni D. Improvement in ischemic parameters during repeated exercise testing: a possible model for myocardial preconditiong. Am J Cardiol. 1996;78:1087-91.

21. Longobardi G, Abete P, Ferrara N, Papa A, Rosiello R, Gurgi G, et al. "Warm-up" phenomenon in adult and elderly patients with coronary artery disease: Further evidence of the loss of "ischemic preconditioning" in the aging heart. J Gerontol A Biol Sci Med Sci. 2000;55:M124-9. 
22. Galinanes M, Fowler AG. Role of clinical pathologies in myocardial injury following ischaemia and reperfusion. Cardiovasc Res. 2004;61:512-21.

23. Salie R, Huisamen B, Lochner A. High carbohydrate and high fat diets protect the heart against ischaemia/reperfusion injury. Cardiovasc Diabetol. 2014;13:109

24. Ferdinandy P, Hausenloy DJ, Heusch G, Baxter GF, Schulz R. Interaction of risk factors, comorbidities, and comedications with ischemia/reperfusion injury and cardioprotection by preconditioning, postconditioning, and remote conditioning. Pharmacol Rev. 2014;66:1142-74.

25. Fryer RM, Auchampach JA, Gross GJ. Therapeutic receptor targets of ischemic preconditioning. Cardiovascular Res. 2002;55:520-5.

26. Van der Mieren G, Nevelsteen I, Vanderper A, Oosterlinck W, Flameng W, Herijgers P. Angiotensin-converting enzyme inhibition and food restriction restore delayed preconditioning in diabetic mice. Cardiovasc Diabetol. 2013;12:36.

27. Clarke SJ, McCormick LM, Dutka DP. Optimising cardioprotection during myocardial ischaemia: targeting potential intracellular pathways with glucagon-like peptide-1. Cardiovasc Diabetol. 2014;13:12.

28. Gross GJ, Peart JN. KATP channels and myocardial preconditioning: an update. Am J Physiol Heart Circ Physiol. 2003;285:H921-30.

29. Garcia RMR, Rezende PC, Hueb W. Impact of hypoglycemic agents on myocardial ischemic preconditioning. World J Diabetes. 2014;5:258-66.

30. Miki T, Itoh T, Sunaga D, Miura T. Effects of diabetes on myocardial infarct size and cardioprotection by preconditioning and postconditioning. Cardiovasc Diabetol. 2012;11:67.

31. Liu Y, Thomton JD, Cohen MV, Downey JM, Schaffer SW. Streptozotocin-induced non-insulin-dependent diabetes protects the heart from infarction. Circulation. 1993;88:1273-8.

32. Honda T, Kaikita K, Tsujita K, Hayasaki T, Matsukawa M, Fuchigami S, et al. Pioglitazone, a peroxisome proliferator-activated receptor-gamma agonist, attenuates myocardial ischemia-reperfusion injury in mice with metabolic disorders. J Mol Cell Cardiol. 2008;44:915-26.

33. Miki T, Miura T, Hotta H, Tanno M, Yano T, Sato T, et al. Endoplasmic reticulum stress in diabetic hearts abolishes erythropoietin-induced myocardial protection by impairment of phospho-glycogen synthase kinase-3beta-mediated suppression of mitochondrial permeability transition. Diabetes. 2009;58:2863-72.

34. Tsang A, Hausenloy DJ, Mocanu MM, Carr RD, Yellon DM. Preconditioning the diabetic heart. The importance of Akt phosphorylation. Diabetes. 2005;54:2360-4.

35. Bilinska M, Potocka J, Korzeniowska-Kubacka I, Piotrowicz R. "Warm-up" phenomenon in diabetic patients with stable angina treated with diet and sulfonylureas. Coron Arstery Dis. 2007;18:455-62.

36. Rezende PC, Garcia RM, Uchida AH, Costa LM, Scudeler TL, Melo RM, et al. Hypotheses, rationale, design, and methods for evaluation of ischemic preconditioning assessed by sequential exercise tests in diabetic and non-diabetic patients with stable coronary artery disease - a prospective study. BMC Cardiovasc Disord. 2013;13:117.

37. American Diabetes Association. Standards of Medical Care in Diabetes - 2015: Summary of Revisions. Diabetes Care. 2015;38:S4.

38. Rahmi RM, Uchida AH, Rezende PC, Lima EG, Garzillo CL, Favarato D, et al. Effect of hypoglycemic agentes on ischemic preconditioning in patients with type 2 diabetes and symptomatic coronary artery disease. Diabetes Care. 2013;36:1654-9.

39. Tomai F, Danesi A, Ghini AS, Crea F, Perino M, Gaspardone A, et al. Effects of K (ATP) channel blockade by glibenclamide on the warm-up phenomenon. Eur Heart J. 1999;20:196-202.

40. Garratt KN, Brady PA, Hassinger NL, Grill DE, Terzic A, Holmes DR. Sulfonylurea drugs increase early mortality in patients with diabetes mellitus after direct angioplasty for acute myocardial infarction. J Am Coll Cardiol. 1999;33:119-24.

41. Ghosh S, Standen NB, Galinãnes M. Failure to precondition pathological human myocardium. J Am Coll Cardiol. 2001;37:711-8.

42. Cleveland Jr JC, Meldrum DR, Cain BS, Banerjee A, Harken AH. Oral sulphonylurea hypoglycaemic agents prevent ischemic preconditioning in human myocardium. Two paradoxes revisited. Circulation. 1997;96:29-32.

43. Su H, Sun X, Ma H, Zhang HF, Yu QJ, Huang C, et al. Acute hyperglycemia exacerbates myocardial ischemia/reperfusion injury and blunts cardioprotective effect of GIK. Am J Physiol Endocrinol Metab. 2007;293:E629-35.

\section{Submit your next manuscript to BioMed Central and take full advantage of:}

- Convenient online submission

- Thorough peer review

- No space constraints or color figure charges

- Immediate publication on acceptance

- Inclusion in PubMed, CAS, Scopus and Google Scholar

- Research which is freely available for redistribution

Submit your manuscript at www.biomedcentral.com/submit 